\title{
CARD9: key player or bystander in cardiac remodeling under hypertension?
}

\author{
Lei $\mathrm{Xi}^{1}$
}

Received: 26 July 2020 / Revised: 30 July 2020 / Accepted: 30 July 2020 / Published online: 2 September 2020

(c) The Japanese Society of Hypertension 2020

N-terminal caspase recruitment domain family member 9 (CARD9) was proposed by Bertin et al. in 2000 to function as an activation domain that mediates homophilic interactions with an upstream CARD-containing activator of nuclear factor $\kappa \mathrm{B}(\mathrm{NF}-\mathrm{\kappa B})$, and CARD9 binds to B-cell lymphoma/leukemia 10 (BCL10) and activates NF- $\mathrm{BB}$ signaling [1]. CARD9 was subsequently identified by a German group in 2006 as a key transducer of Dectin-1 signaling, which is the major mammalian pattern recognition receptor for the fungal component zymosan [2]. CARD9 controls Dectin-1-mediated myeloid cell activation, cytokine production, and innate antifungal immunity. In 2007, researchers from The M.D. Anderson Cancer Center generated CARD9 homozygous knockout mice and demonstrated that CARD9-deficient macrophages had defects in the activation of the kinases p38 and JNK but not NF- $\kappa B$ after bacterial or viral infection [3]. CARD9deficient mice also failed to clear infection and showed altered cytokine production after challenge with Listeria monocytogenes. Therefore, there has been a consensus that CARD9 plays an indispensable role in innate immune responses to intracellular pathogens and in regulating several key cytokines [4].

In addition to fungal infection, CARD9 is also involved in tuberculosis infection. A decade ago, Dorhoi et al. reported that CARD9(-/-) mice succumbed early after aerosol infection, with increased mycobacterial burden, pyogranulomatous pneumonia, accelerated granulocyte recruitment, and increased abundance of proinflammatory cytokines in the serum and lungs [5]. CARD9 deficiency had no apparent effect on $\mathrm{T}$ cell responses but had a marked

Lei Xi

lei.xi@vcuhealth.org

1 Pauley Heart Center, Division of Cardiology, Department of Internal Medicine, Virginia Commonwealth University, Richmond, VA 23298-0204, USA impact on the hematopoietic compartment. CARD9(-/-) granulocytes failed to produce IL-10 after Mycobacterium tuberculosis infection, suggesting that the absence of an anti-inflammatory feedback loop accounted for the granulocyte-dominated pathology, uncontrolled bacterial replication, and, ultimately, death of infected CARD9 $(-/-)$ mice and indicating a pivotal role of CARD9 signaling in the autonomous innate host defense against tuberculosis [5]. The same research group also identified the CARD9BCL10 module as an essential component of the RIG-Idependent proinflammatory response and established RIG-I as a sensor for activating inflammasomes in response to certain RNA viruses [6]. Indeed, evidence has shown that human CARD9 deficiency results in selective defects in the host defense against invasive fungal infection and impaired neutrophil killing [7].

However, one may wonder why and how CARD9, a signaling molecule that is essential for bacterial and viral pathogen infection, would significantly affect the cardiac remodeling process and heart function. This issue was first addressed by Ren et al. in 2011, who reported evidence suggesting that CARD9 played an important role in regulating cardiac inflammation and fibrosis in response to elevated angiotensin II (Ang II) [8]. That group found that wild-type mice that received Ang II infusion $(1500 \mathrm{ng} / \mathrm{kg} /$ min for 7 days) had a marked increase in CARD9(+) macrophages in the heart, whereas CARD9 $(-/-)$ mice showed significantly suppressed macrophage infiltration and expression of proinflammatory cytokines, including interleukin-1 $\beta$ (IL-1 $\beta$ ) and connective tissue growth factor, along with diminished cardiac fibrosis markers. Ang IIinduced activation of NF- $\mathrm{kB}$, JNK, and p38 mitogenactivated protein kinase was reduced in CARD9 $(-/-)$ macrophages [8].

In this context, in the current issue of Hypertension Research, Peterson et al. from the University of Wyoming [9] demonstrated a role of CARD9 in pressure overloadinduced pathological cardiac remodeling and dysfunction by means of CARD9-knockout mice subjected to transverse 
aortic constriction (TAC). This experienced investigative team has focused on CARD9 in various cardiovascular diseases, although the current work is the first report on the role of CARD9 in hypertension-related cardiac remodeling. Although cardiomyocyte contractility was modified in cardiomyocytes isolated from CARD9-knockout mice, CARD9 was not expressed in cardiomyocytes. Therefore, the observed detrimental effects of CARD9 on the myocardium were likely mediated by its expression in immune cells, particularly macrophages, residing in or infiltrating the myocardium. Another interesting finding of this study is that the fibrotic areas were reduced in CARD9-knockout hearts even under nonstressed control conditions [9], indicating that mild fibrosis may develop with the aging process, and/or resident immune cells expressing CARD9 were present in the myocardium even under nonstressed conditions and that CARD9 may be involved in the maintenance of collagen turnover in healthy hearts.

It is notable that this research group has previously demonstrated that CARD9-mediated proinflammatory signaling associated with obesity resulting from chronic intake of a high-fat diet (HFD, 45\% fat for 5 months) [10]. CARD9 knockout alleviated HFD-induced insulin resistance and glucose intolerance and prevented myocardial dysfunction with preserved cardiac ejection fraction (HFpEF) and cardiomyocyte contractile properties. The loss of CARD9 also significantly decreased the number of infiltrating macrophages in the heart and reduced myocardium-, plasma-, and macrophage-derived cytokines (i.e., IL-6, IL-1 $\beta$, and TNF $\alpha$ ) and restored cardiac autophagy signaling [10]. This group proposed that systemic inflammation induced macrophage infiltration and activation and the release of cytokines and chemokines, causing a vicious cycle of tissue dysfunction via a positive feedback loop that was CARD9-dependent [11]. Another recent study from these researchers demonstrated that CARD9 knockout protected against myocardial ischemia/reperfusion (I/R) injury by reducing neutrophil infiltration and attenuating CARD9-associated acute inflammatory signaling [12]. Following I/R, CARD9-knockout mice had significantly smaller infarct size and lower numbers of infiltrated neutrophils than wild-type controls.

Interestingly, in contrast, a recent paper reported that CARD9 protected cardiomyocytes from apoptosis by interacting with Apaf-1 and interfering with apoptosome formation following myocardial $\mathrm{I} / \mathrm{R}$ injury in vivo and in vitro [13]. These researchers showed that the loss of CARD9 significantly increased caspase-3 activation and cell death following oxidative stress in vitro and that CARD9 overexpression decreased apoptosis by suppressing caspase-3 activation. CARD9 also attenuated caspase-9 activation by interacting with Apaf-1 via its CARD domain. CARD9(-/-) mice exhibited significantly higher numbers of apoptotic cells after myocardial I/R injury than wild-type mice. Conversely, these new discrepant results undoubtedly add more complexity and confusion regarding the opposing protective or detrimental roles of CARD9 in cardiac diseases proposed by various groups [9, 12, 13].

Another Dutch/German study evaluated whether hematopoietic deletion of Dectin-2 or CARD9 reduced inflammation and atherosclerosis development [14]. Low-density lipoprotein receptor-knockout mice were transplanted with bone marrow from wild-type, Dectin-2-, or CARD9knockout mice and fed a Western diet for 10 weeks. Hematopoietic deletion of CARD9 did not influence plasma triglyceride and cholesterol levels. Unexpectedly, hematopoietic deletion of CARD9 increased atherosclerotic lesion formation and lesion severity without affecting the circulating immune cell composition or peripheral cytokine secretion. These results suggested that the presence of CARD9 protected against the initiation of atherosclerosis development.

The relevance of the TAC model to hypertensive heart disease is also debatable, since there was no increase in peripheral blood pressure in the TAC model in the present study. However, the TAC rodent model was developed in several U.S. laboratories around the year 1994 [15, 16] and has gained popularity around the world as an experimental tool for studying the response of the heart to the increased afterload that commonly occurs during chronic hypertension. Other reasonable concerns may include critiques of the imperfect experimental model using global CARD9knockout mice. However, considering that there are no specific chemical inhibitors or activators of CARD9 currently available, the use of CARD9-knockout mice remains useful, if not the only tool to investigate the cause-effect relationship between CARD9 and cardiovascular pathologies such as hypertension and cardiac remodeling. Peterson et al. used CARD9-/- breeders originally generated by Xin Lin's group in 2007 [3]. CARD9 was mainly expressed in macrophages and myeloid dendritic cells and in organs abundant in myeloid cells such as the thymus, spleen, bone marrow, lungs, and lymph nodes. The degree of expression of CARD9 in regular mouse myeloid cells could potentially support a role for CARD9 in TAC-induced cardiac maladaptation. Although it is uncertain whether increased expression of CARD9 is sufficient to cause TAC-related abnormalities, this study presented evidence suggesting that CARD9 is necessary for pathological cardiac remodeling in response to the increased cardiac afterload induced by TAC. Alternative models such as bone marrow transplantation and cardiac-specific knockout of CARD9 may be helpful. However, these models cannot overcome the fact that CARD9 expression in the myocardium is limited to resident and infiltrating myeloid cells, and CARD9 is not expressed by cardiomyocytes. 


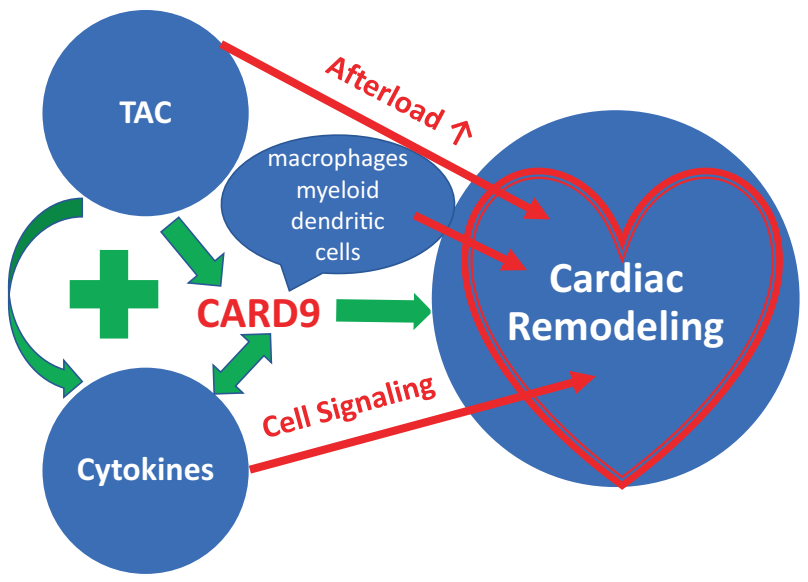

Fig. 1 Schematic summary of the mechanistic pathways of CARD9regulated cardiac remodeling following TAC. Abbreviations: CARD9 $\mathrm{N}$-terminal caspase recruitment domain family member 9, TAC transverse aortic constriction

In conclusion, the present study by Peterson et al. [9] has provided another solid piece of evidence indicating that CARD9 is likely a key player and not a bystander in cardiac remodeling under hypertension (summarized in Fig. 1). However, the exact mechanism of the mediators of blood-heart cell communication and signal transduction remain to be further elucidated in the future. CARD9 may eventually become a valid target for pharmaceutical development in finding better drugs to treat cardiovascular diseases.

\section{Compliance with ethical standards}

Conflict of interest The author declares no conflict of interest.

Publisher's note Springer Nature remains neutral with regard to jurisdictional claims in published maps and institutional affiliations.

\section{References}

1. Bertin J, Guo Y, Wang L, Srinivasula SM, Jacobson MD, Poyet JL, et al. CARD9 is a novel caspase recruitment domaincontaining protein that interacts with BCL10/CLAP and activates NF-kappa B. J Biol Chem. 2000;275:41082-6.

2. Gross O, Gewies A, Finger K, Schafer M, Sparwasser T, Peschel $\mathrm{C}$, et al. Card9 controls a non-TLR signalling pathway for innate anti-fungal immunity. Nature. 2006;442:651-6.
3. Hsu YM, Zhang Y, You Y, Wang D, Li H, Duramad O, et al. The adaptor protein CARD9 is required for innate immune responses to intracellular pathogens. Nat Immunol. 2007;8:198-205.

4. Roth S, Ruland J. Caspase recruitment domain-containing protein 9 signaling in innate immunity and inflammation. Trends Immunol. 2013;34:243-50.

5. Dorhoi A, Desel C, Yeremeev V, Pradl L, Brinkmann V, Mollenkopf HJ, et al. The adaptor molecule CARD9 is essential for tuberculosis control. J Exp Med. 2010;207:777-92.

6. Poeck H, Bscheider M, Gross O, Finger K, Roth S, Rebsamen M, et al. Recognition of RNA virus by RIG-I results in activation of CARD9 and inflammasome signaling for interleukin 1 beta production. Nat Immunol. 2010;11:63-9.

7. Drewniak A, Gazendam RP, Tool AT, van Houdt M, Jansen MH, van Hamme JL, et al. Invasive fungal infection and impaired neutrophil killing in human CARD9 deficiency. Blood. 2013;121:2385-92.

8. Ren J, Yang M, Qi G, Zheng J, Jia L, Cheng J, et al. Proinflammatory protein CARD9 is essential for infiltration of monocytic fibroblast precursors and cardiac fibrosis caused by Angiotensin II infusion. Am J Hypertens. 2011;24:701-7.

9. Peterson MR, Getiye Y, Bosch L, Sanders AJ, Smith AR, Haller $\mathrm{S}$, et al. A potential role of caspase recruitment domain family member 9 (Card9) in transverse aortic constriction-induced cardiac dysfunction, fibrosis, and hypertrophy. Hypertens Res. 2020. https://doi.org/10.1038/s41440-020-0507-0.

10. Cao L, Qin X, Peterson MR, Haller SE, Wilson KA, Hu N, et al. CARD9 knockout ameliorates myocardial dysfunction associated with high fat diet-induced obesity. J Mol Cell Cardiol. 2016;92:185-95.

11. Peterson MR, Haller SE, Ren J, Nair S, He G. CARD9 as a potential target in cardiovascular disease. Drug Des Devel Ther. 2016;10:3799-804.

12. Qin X, Peterson MR, Haller SE, Cao L, Thomas DP, He G. Caspase recruitment domain-containing protein 9 (CARD9) knockout reduces regional ischemia/reperfusion injury through an attenuated inflammatory response. PLoS ONE. 2018;13: e0199711. https://doi.org/10.1371/journal.pone.0199711.

13. Li Y, Liang P, Jiang B, Tang Y, Liu X, Liu M, et al. CARD9 promotes autophagy in cardiomyocytes in myocardial ischemia/ reperfusion injury via interacting with Rubicon directly. Basic Res Cardiol. 2020;115:29. https://doi.org/10.1007/s00395-0200790-6.

14. Thiem K, Hoeke G, van den Berg S, Hijmans A, Jacobs CWM, Zhou E, et al. Deletion of hematopoietic Dectin-2 or CARD9 does not protect against atherosclerotic plaque formation in hyperlipidemic mice. Sci Rep. 2019;9:4337. https://doi.org/10.1038/ s41598-019-40663-x.

15. Rockman HA, Wachhorst SP, Mao L, Ross J Jr. ANG II receptor blockade prevents ventricular hypertrophy and ANF gene expression with pressure overload in mice. Am J Physiol. 1994;266:H2468-75.

16. Dorn GW 2nd, Robbins J, Ball N, Walsh RA. Myosin heavy chain regulation and myocyte contractile depression after LV hypertrophy in aortic-banded mice. Am J Physiol. 1994;267:H400-5. 